\title{
Características produtivas de cultivares de batata-doce sob condições irrigadas e de sequeiro na região norte de Minas Gerais.
}

\author{
Geraldo M. de Resende \\ Embrapa Semi-Árido, C. Postal 23, 56.300-000 Petrolina-PE e-mail: gmilanez@cpatsa.embrapa.br
}

RESUMO

Com o objetivo de selecionar cultivares de batata-doce em condições de irrigação suplementar e de sequeiro, instalou-se dois ensaios no Campo Experimental do Gorutuba em Porteirinha-MG, de novembro de 1990 a abril de 1991. O delineamento experimental foi de blocos ao acaso com oito cultivares (Brazlândia Branca, Brazlândia Rosada, Brazlândia Roxa, Coquinho, Princesa, Arroba, Rama Roxa e Paulistinha) e cinco repetições. Sob irrigação suplementar, destacou-se a cultivar Brazlândia Branca com maior rendimento (22,3 t/ha), seguida das cultivares Paulistinha (21,3 t/ha) e Princesa (19,0 t/ha), que não mostraram diferenças significativas entre si. A cultivar Brazlândia Roxa apresentou o menor desempenho (13,5 t/ha), assim como a maior produção de refugo $(7,2 \mathrm{t} / \mathrm{ha})$. Para peso médio de raiz houve uma variação de 219,9 a 337,6g, sobressaindo-se a cultivar Brazlândia Rosada $(337,6$ g). Nas condições de sequeiro, a cultivar Paulistinha destacou-se, com 17,6 t/ha, seguida das cultivares Princesa (12,3 t/ha), Brazlândia Branca (10,9 t/ha) e Rama Roxa (10,2 t/ha), não havendo diferença significativa entre as três últimas. $\mathrm{O}$ menor rendimento $(8,2 \mathrm{t} / \mathrm{ha})$, assim como o maior refugo (6,9 t/ha), foram apresentados pela cultivar Brazlândia Roxa. O peso médio de raiz, nas condições de sequeiro, oscilou de 124,4 g (Brazlândia Rosada), sobressaindo-se a cultivar Paulistinha com $249,9 \mathrm{~g}$.

Palavras-Chave: Ipomoea batatas, rendimento comercial, refugo, desempenho.

\begin{abstract}
Productive characteristics of sweet potato cultivars under irrigated and dryland conditions in the north region of the State of Minas Gerais.

Two experiments were carried out at the Gorutuba Experimental Field, Porteirinha-MG, Brazil, from November 1990 to April 1991, to evaluate sweet potato cultivars under irrigated and dryland conditions. The experimental design was a complete randomised block with eight cultivars (Brazlândia Branca, Brazlândia Rosada, Brazlândia Roxa, Coquinho, Princesa, Arroba, Rama Roxa and Paulistinha) and five replications. Under irrigated conditions, the cultivars Brazlândia Branca showed the highest yield (22.3 t/ha) followed by Paulistinha (21.3 t/ ha) and Princesa (19.0 t/ha) with no significant differences among them. The cultivar Brazlândia Roxa showed the lowest yield (13.5 t/ ha) as well as the highest non-commercial yield (7.2 t/ha). Mean root weight under irrigation, varied from $219.9 \mathrm{~g}$ (Brazlândia Roxa) to $337.6 \mathrm{~g}$ (Brazlândia Rosada). Under dryland conditions, the cultivar Paulistinha showed the highest yield (17.6 t/ha), followed by Princesa (12.3 t/ha), Brazlândia Branca (10.9 t/ha) and Rama Roxa (10.2 t/ha), with no significant differences among the last three cultivars. The lowest commercial yield ( $8.2 \mathrm{t} / \mathrm{ha})$ and the highest non-commercial yield (6.9 t/ha) were showed by the cultivar Brazlândia Roxa. Root mean weight under dryland conditions varied from $124.4 \mathrm{~g}$ (Brazlândia Roxa) to $249.9 \mathrm{~g}$ (Paulistinha).
\end{abstract}

Keywords: Ipomoea batatas, commercial yield, non-commercial yield, performance.

\section{(Aceito para publicação em 07 de abril de 1999)}

\begin{abstract}
A batata-doce é uma hortaliça de grande importância social e econômica vislumbrando-se sua participação efetiva no suprimento de alimentos. Além disso constitui-se numa excelente alternativa para alimentação animal e para a agroindústria (Thomazelli et al., 1997), apresentando um rendimento nacional de 11,31 t/ha (ANUÁRIO, 1996). É cultura de clima quente, necessitando de temperaturas noturnas mais frias (20 ${ }^{\circ} \mathrm{C}$ ) e fotoperíodo longo para o desenvolvimento das raízes (IITA, 1993).

Quanto à sua capacidade produtiva, há uma série de autores que relatam diferentes rendimentos para a cultura. $\mathrm{O}$ rendimento se situa de 25 a 30 t/ha (Miranda et al., 1989), varia de 10 a 15 t/ha (Camargo, 1992) e oscila entre 15 a 18 t/ha (Kurihara et al., 1993).
\end{abstract}

A batata-doce produz bem em regiões com 750 a $1000 \mathrm{~mm}$ anuais de chuva, com cerca de 500 a $600 \mathrm{~mm}$ bem distribuídos durante os cinco meses de ciclo da cultura (Miranda et al., 1989), sendo suficiente um regime de chuvas que garanta 500 a $700 \mathrm{~mm}$ bem distribuídos durante seu ciclo (EMPASC/ ACARESC, 1991). Para as condições de Goiás, Peixoto \& Miranda (1984), informam que nos plantios realizados de outubro a dezembro, as necessidades hídricas da cultura são geralmente supridas pelas chuvas, sendo que até o pegamento das mudas, o solo deve estar sempre úmido. Peterson citado por King (1985), relata que o suprimento de $25 \mathrm{~mm}$ de água por semana, resultou em maior rendimento, comparado a aplicação de lâmina de água de $12 \mathrm{~mm}$. A apli- cação de $25 \mathrm{~mm}$ de água por semana equivale a um total de $500 \mathrm{~mm}$ por um período de cinco meses de ciclo. Aplicando uma lamina de $10 \mathrm{~mm}$ em irrigações a cada dois dias, com plantio em novembro e colheita em maio (seis meses), Cecílio Filho et al. (1998) verificaram maior rendimento para cultivar Brazlândia Branca (20,50 t/ha).

Avaliando oito clones de batata-doce em doze condições ambientais, Martin et al. (1988) verificaram rendimentos de 20,3 a 37,3 t/ha com colheita aos 135 dias após plantio, irrigando-se de acordo com a necessidade, durante o desenvolvimento da cultura. Testando doze clones de batata-doce, sob irrigação por aspersão e colheita aos 152 dias, Peixoto et al. (1989) observaram rendimentos variando de 16,7 a 27,0 t/ha. A cul- 
Tabela 1. Valores mensais de precipitação pluviométrica acumulada $(\mathrm{mm})$, temperatura do ar $\left({ }^{\circ} \mathrm{C}\right)$ e umidade relativa do ar (\%) média durante a execução dos experimentos. Porteirinha - MG, EPAMIG, 1991.

\begin{tabular}{lccc}
\hline Meses & Precipitação & Temperatura & $\begin{array}{c}\text { Umidade } \\
\text { relativa }\end{array}$ \\
\hline Novembro & 23,2 & 25,1 & 83,0 \\
Dezembro & 206,2 & 26,4 & 72,1 \\
Janeiro & 202,3 & 25,8 & 78,2 \\
Fevereiro & 54,2 & 27,0 & 74,4 \\
Março & 65,3 & 25,1 & 74,0 \\
Abril & 23,2 & 26,0 & 70,7 \\
\hline Total & 574,4 & - & - \\
\hline
\end{tabular}

tivar Brazlândia Branca apresentou 26,7 t/ha, seguida pela Princesa (26,6 t/ha), Brazlândia Rosada (25,8 t/ha), Coquinho (18,1 t/ha) e Brazlândia Roxa $(17,7$ t/ha). A produção de refugo oscilou entre 1,6 e 4,5 t/ha, tendo a cultivar Brazlândia Roxa se destacado com 4,5 t/ha. O peso médio de raízes variou de 183,3 g (Brazlândia Roxa) a 232,1 g (Brazlândia Rosada).

Estudando espaçamentos, Mendonça \& Peixoto (1991) verificaram que com o espaçamento de $0,80 \times 0,40 \mathrm{~m}$, as cultivares Brazlândia Branca e Brazlândia Roxa apresentaram rendimento de 20,21 e 19,54 t/ha e peso médio de raiz de 220,08 e 197,54 g, respectivamente, com ciclo de 152 dias e plantio no período chuvoso.

O presente trabalho teve como objetivo avaliar o comportamento de cultivares regionais e introduzidas de batata-doce em condições de sequeiro (período chuvoso) e com irrigação suplementar nas condições da região Norte de Minas Gerais.

\section{MATERIAL E MÉTODOS}

Dois experimentos, um com irrigação suplementar e outro em condições de sequeiro foram conduzidos no Campo Experimental do Gorutuba, da Empresa de Pesquisa Agropecuária de $\mathrm{Mi}$ nas Gerais (EPAMIG), em PorteirinhaMG, no período de novembro de 1990 a abril de 1991, em solo aluvião, textura arenosa. A análise de solo apresentou as seguintes características químicas: $\mathrm{K}=0,12 \mathrm{cmol}_{\mathrm{c}} \mathrm{dm}^{-3}, \mathrm{P}=80 \mathrm{mg} \mathrm{dm}$ 3; $\mathrm{Ca}=2,5 \mathrm{cmol}_{\mathrm{c}} \mathrm{dm}^{-3} ; \mathrm{Mg}=0,9 \mathrm{cmol}_{\mathrm{c}}$ $\mathrm{dm}^{-3} ; \mathrm{H}+\mathrm{Al}=0,1 \mathrm{cmol}_{\mathrm{c}} \mathrm{dm}^{-3} ; \mathrm{pH} \mathrm{em} \mathrm{H}_{2} \mathrm{O}$ $=6,1 ;$ areia $=850 \mathrm{~g} \mathrm{~kg}^{-1} ;$ silte $=100 \mathrm{~g}$ $\mathrm{kg}^{-1}$; argila $=50 \mathrm{~g} \mathrm{~kg}^{-1}$ e matéria orgâni$\mathrm{ca}=8 \mathrm{~g} \mathrm{~kg}^{-1}$. Os dados climátologicos durante a execução dos experimentos

$\mathrm{O}$ delineamento experimental foi de blocos ao acaso com oito cultivares (introduzidas: Brazlândia Branca, Brazlândia Rosada, Brazlândia Roxa, Coquinho e Princesa, oriundas do Centro Nacional de Pesquisa de Hortaliças) e regionais: Paulistinha, Arroba e Rama Roxa) com cinco repetições. As parcelas constituíram-se de quatro fileiras de $4,5 \mathrm{~m}$ de comprimento espaçadas de $0,80 \mathrm{~m}$ e 0,30 $\mathrm{m}$ entre plantas, sendo usadas como área útil as duas fileiras centrais. A adubação constou de $330 \mathrm{~kg} / \mathrm{ha}$ de superfosfato simples e $50 \mathrm{~kg} / \mathrm{ha}$ de cloreto de potássio, incorporados no sulco de plantio.

Nos plantios (28/11/90) foram utilizadas mudas (ramos) com oito entrenós, ficando enterrados três a quatro nós, colocados transversalmente sobre a leira, a uma profundidade de $10-15 \mathrm{~cm}$. A cultura foi mantida no limpo, através de capinas manuais, não sendo realizados quaisquer tratos fitossanitários.

No experimento de sequeiro a precipitação no período foi de $574,4 \mathrm{~mm}$ (Tabela 1), sendo que no experimento com irrigação suplementar, foram necessárias três irrigações complementares através de sulcos de infiltração, nos meses de dezembro, janeiro e fevereiro com lâminas de 40 mm, que somadas à precipitação do período, acumularam um total de 694,4 mm durante o ciclo.

As colheitas foram realizadas 150 dias após o plantio, sendo avaliadas as são apresentados na Tabela 1. seguintes características: rendimento comercial (raízes com peso acima de $80 \mathrm{~g}$ ), refugo (raízes abaixo de $80 \mathrm{~g}$, rachadas, deformadas, esverdeadas, brocadas e com veias) e peso médio de raiz comercial. Posteriormente, utilizandose o programa computacional SAS (Statistical Analysis System Institute, 1989) realizou-se a análise de variância conjunta dos experimentos para cada característica avaliada, aplicando-se o teste de Tukey a 5\% de probabilidade para comparação das médias.

\section{RESULTADOS E DISCUSSÃO}

Os resultados evidenciaram efeitos significativos para experimentos e para a interação experimentos $\mathrm{x}$ cultivares para as características avaliadas.

Para o experimento com irrigação suplementar (Tabela 2), destacou-se quanto a rendimento comercial a cultivar Brazlândia Branca (22,3 t/ha), seguida das cultivares Paulistinha (21,3 t/ ha) e Princesa (19,0 t/ha), que não mostraram diferenças significativas entre si. O pior desempenho foi apresentado pela cultivar Brazlândia Roxa (13,5 t/ha), porem não mostrando diferenças significativas das cultivares Coquinho, Rama Roxa, Arroba e Brazlândia Rosada. Esses resultados concordam com os obtidos por Cecílio Filho et al.(1998), estando acima do rendimento médio nacional de 11,31 t/ha (ANUÁRIO, 1996). Assim como situam-se nas faixas de rendimento para a cultura relatadas por Camargo (1992) e Kurihara et al. (1993), sendo no entanto, inferiores ao rendimento de 25 a 30 t/ha informado por Miranda et al. (1989) e Miranda (1989). O baixo desempenho da cultivar Brazlândia Roxa (13,5 t/ha) devese, provavelmente, ao período de 150 dias ter sido insuficiente para seu pleno desenvolvimento vegetativo, resultando em maior rendimento de raízes refugos (7,2 t/ha) e sobretudo de raízes com peso abaixo de $80 \mathrm{~g}$. Estes resultados são similares aos encontrados por Peixoto et al. (1989), que também verificaram ser a cultivar Brazlândia Roxa mais tardia e que, quando colhida aos 152 dias, apresentou o maior rendimento de refugo. No que se refere ao experimento em condições de sequeiro, verificou-se um 
Tabela 2. Rendimento comercial e de refugo e peso médio de raízes de cultivares de batata-doce em condições de sequeiro e irrigação suplementar. Porteirinha-MG, EPAMIG, 1990/91.

\begin{tabular}{|c|c|c|c|c|c|c|}
\hline \multirow{3}{*}{$\frac{\text { Cultivares }}{\text { Braz. Branca }}$} & \multicolumn{2}{|c|}{ Rendimento (t/ha) } & \multicolumn{2}{|c|}{ Refugo (t/ha) } & \multicolumn{2}{|c|}{ Peso médio/raízes (g) } \\
\hline & \multirow{2}{*}{$\frac{\mid \text { Irrigado }}{22,3 a^{*}}$} & Sequeiro & \multirow{2}{*}{$\begin{array}{c}\text { Irrigado } \\
4,4 \mathrm{~b}\end{array}$} & \multirow{2}{*}{$\begin{array}{c}\text { Sequeiro } \\
4,0 \mathrm{~b}\end{array}$} & \multirow{2}{*}{\begin{tabular}{|l|} 
Irrigado \\
$277,5 \mathrm{ab}$
\end{tabular}} & Sequeiro \\
\hline & & 10,9 bc & & & & $164,5 \mathrm{bcd}$ \\
\hline Paulistinha & $21,3 a b$ & $17,6 \mathrm{a}$ & $2,9 \mathrm{bc}$ & 3,8 b & $258,0 \quad b$ & 249,9 a \\
\hline Princesa & $19,0 \mathrm{abc}$ & $12,3 \mathrm{~b}$ & $3,2 \mathrm{bc}$ & $3,5 \mathrm{bc}$ & 256,1 & 191,5 bc \\
\hline Braz. Rosada & $16,7 \mathrm{bcd}$ & 9,1 & $3,7 \mathrm{bc}$ & $2,3 \mathrm{bc}$ & 337,6 a & $199,2 \mathrm{abc}$ \\
\hline Arroba & $16,5 \mathrm{bcd}$ & 8,3 & $4,1 \mathrm{bc}$ & $3,4 \mathrm{bc}$ & $246,4 \quad b$ & $149,7 \quad c d$ \\
\hline Rama Roxa & $16,1 \mathrm{bcd}$ & $10,2 \mathrm{bcd}$ & $2,8 \quad c$ & 3,2 bc & 253,1 & 163,8 \\
\hline Coquinho & 14,9 & 9,5 & $3,8 \mathrm{bc}$ & $3,7 \mathrm{bc}$ & 273,2 & $213,2 a b$ \\
\hline Braz. Roxa & 13,5 & 8,2 & $7,2 \mathrm{a}$ & $6,9 \mathrm{a}$ & $219,9 \quad b$ & 124,4 \\
\hline Médias & $17,5 \mathrm{~A}$ & $10,8 \mathrm{~B}$ & $4,0 \mathrm{~A}$ & $3,8 \mathrm{~A}$ & 265,2 A & $182,0 \mathrm{~B}$ \\
\hline C.V. (\%) & & & & & & \\
\hline
\end{tabular}

*Médias seguidas pela mesma letra minúscula nas colunas e maiúsculas nas linhas, não diferem entre si, pelo teste de Tukey a 5\% de probabilidade.

rendimento comercial variando de 8,2 a 17,6 t/ha (Tabela 2). A cultivar Paulistinha apresentou o maior rendimento (17,6 t/ha), seguida pelas cultivares Princesa (12,3 t/ha), Brazlândia Branca (10,9 t/ha) e Rama Roxa (10,2 t/ ha) que não mostraram diferenças significativas entre si. Os resultados obtidos pelas cultivares mais produtivas estão acima da média nacional de 11,31 t/ ha (ANUÁRIO, 1996), estando dentro das variações de rendimento relatadas por Camargo (1992), que informa rendimentos de 10 a 15 t/ha e por Peixoto et al. (1989), que colhendo aos 146 dias, observaram rendimentos oscilando de 7,3 a 16,7 t/ha. Resultados similares ao experimento irrigado foram obtidos pela cultivar Brazlândia Roxa em condições de sequeiro. $\mathrm{O}$ menor rendimento comercial $(8,2 \mathrm{t} / \mathrm{ha})$, associado ao maior de refugos $(6,9 \mathrm{t} / \mathrm{ha})$, sugere a necessidade de se colher esta cultivar num período superior a 150 dias.

Com relação ao peso médio de raízes, verificou-se uma variação de 219,9 g a 337,6 g (Tabela 2) sobressaindo-se a cultivar Brazlândia Rosada (337,6 g), seguida da Brazlândia Branca $(277,5 \mathrm{~g})$, não diferindo entre si. Estes resultados são divergentes dos encontrados por Peixoto et al. (1989) e Mendonça \& Peixoto (1991), estando no entanto, com o tamanho ideal para o comércio (200 a 400 g), de acordo com Miranda (1989). Quanto ao peso médio de raízes em condições de sequeiro (Tabela 2), verificou-se uma oscilação de 124,4 g a 249,9 g, destacando-se a cultivar Paulistinha $(249,9 \mathrm{~g})$, seguida pelas cultivares Coquinho $(213,2 \mathrm{~g})$ e Brazlândia Rosada $(199,2 \mathrm{~g})$ que não mostraram diferenças entre si. Esses resultados são similares aos encontrados por Peixoto et al. (1989), que colhendo aos 146 dias após o plantio, verificaram uma variação de 103,4 g a 189,9 g para peso médio de raízes, sendo o pior desempenho apresentado pela cultivar Brazlândia Roxa (103,4 g).

Comparando-se os experimentos (Tabela 2) verificou-se que o maior rendimento médio foi apresentado pelo experimento com irrigação suplemen$\operatorname{tar}(17,5 \mathrm{t} / \mathrm{ha})$, assim como o maior peso médio de raiz (265,2 g), não havendo diferenças estatísticas para o rendimento de raízes não comerciais (refugo). $\mathrm{O}$ menor rendimento do experimento de sequeiro (10,8 t/ha) deve-se a má distribuição das chuvas, que ocorreram na sua maioria, no início do ciclo, nos primeiros 60 dias $(75,2 \%)$ o que promoveu um bom desenvolvimento vegetativo inicial, comprometendo contudo, uma bom acúmulo de reservas pela menor ocorrência de chuvas $(20,8 \%)$ no período de fevereiro/março (Tabela 1). Segundo Miranda et al. (1989), um regime de chuvas com 750 a $1000 \mathrm{~mm}$ anuais, com cerca de 500 a $600 \mathrm{~mm}$ bem distribuídos durante o ciclo da cultura, proporciona um bom rendimento da cultura. Neste sentido, o experimento com irrigação suplementar (694,4 mm) atendeu às necessidades da cultura.

Salienta-se, ainda, com relação ao rendimento de raízes não comerciais, que a sua grande maioria deveu-se, principalmente, a raízes abaixo do padrão (peso abaixo de $80 \mathrm{~g}$ ), uma vez que durante a execução dos experimentos não verificou-se ataque de pragas de solo, um dos principais fatores que promovem um maior rendimento de refugos.

Os resultados obtidos permitem indicar como orientação geral para uso dos produtores em condições irrigadas as cultivares Brazlândia Branca, Princesa e Brazlândia Rosada, introduzidas da Embrapa Hortaliças e a própria cultivar Paulistinha. Para as condições de sequeiro recomenda-se aos produtores de batata-doce as cultivares Paulistinha, Princesa e Brazlândia Branca. Assim como de forma geral a cultivar Paulistinha se mostrou com ampla capacidade de adaptação, comportando-se bem tanto em condições de sequeiro quando nas condições irrigadas, provavelmente em razão de ser um material adaptado às condições regionais onde foram realizados os trabalhos. Por esta razão os resultados desse estudo consolidam a cultivar Paulistinha para a região além de indicar como nova alternativa as cultivares introduzidas da Embrapa. Assim como, verificou-se a 
necessidade de se realizar mais pesquisas com a cultivar Brazlândia Roxa em relação ao ciclo da cultivar, uma vez que a mesma, além de um excelente aspecto comercial, apresenta boa resistência a insetos de solo (Miranda et al., 1989), como também ao nematóide Meloidogyne javanica (Silveira \& Maluf, 1993).

\section{LITERATURA CITADA}

ANUÁRIO ESTATÍSTICO DO BRASIL. Rio de Janeiro: IBGE, v. 56, 1996. p. 3-42.

CAMARGO, L. de S. As hortaliças e seu cultivo. 3.ed., Campinas: Fundação Cargill, 1992. 252 p.

EMPASC/ACARESC. Normas técnicas para a cultura da batata-doce; Santa Catarina. Florianóplis: EMPASC/ACARESC, 1991. 21 p. (EMPASC/ACARESC. Sistemas de produção, 15).

CECÍLIO FILHO, A.B.; REIS, M. dos S.; SOUZA, R.J. de; PASQUAL, M. Degenerescência em cultivares de batata-doce. Horticultura Brasileira, Brasília, v. 16, n. 1, p. 82-84, 1998.
INTERNATIONAL INSTITUTE OF TROPICAL AGRICULTURE (IITA). Sustainable food production in sub Saharan - Africa - 2. Constraints and opportunities. Ibadan: IITA, 1993. $108 \mathrm{p}$.

KING, G.A. The effect of time of planting on yield of six varieties of sweet potato (Ipomoea batatas (L) Lam.) in southern costal lowlands of Papua New Guinea. Tropical Agriculture, v. 62, n. 3, p. 225-228, 1985.

KURIHARA, C.; GOMES, G.C.; MATOS, F.A.C.; QUINDERÉ JUNIOR, R.A.G. Recomendações técnicas para a produção e comercialização de hortaliças para o período de entressafra no Distrito Federal. Brasília: EMBRAPA/SPI/EMATER-DF, 1993. 43 p.

MARTIN, F.W.; FLORES, N.A.; CARMER, S.G. Identification of a key enviroment for determination of yield stability in sweet potato. Tropical Agriculture, v. 65, n. 4, p. 313-316, 1988.

MENDONÇA, A.T.C.; PEIXOTO, N. Efeitos do espaçamento e de níveis de adubação em cultivares de batata-doce. Horticultura Brasileira, Brasília, v. 9, n. 2, p. 80-82, 1991.

MIRANDA, J.E.C. de. Brazlândia Roxa, Brazlândia Branca, Brazlândia Rosada e Coquinho: novas cultivares de batata-doce. Horticultura Brasileira, Brasília, v. 7, n. 1, p. 32-33, 1989.
MIRANDA, J.E.C. de; FRANÇA, F.H.; CARRIJO, O.A.; SOUZA A.F.; PEREIRA, W.; LOPES, C.A. Batata-doce (Ipomoea batatas (L) Lam). Brasília; EMBRAPA/CNPH, 1989. 20 p. (EMBRAPA. Circular Técnica, 3).

PEIXOTO, N.; MIRANDA, J.E.C. de. $O$ cultivo da batata doce em Goiás. Goiânia: EMGOPADDI, 1984. 24 p. (EMGOPA. Circular Técnica, 7).

PEIXOTO, N., MIRANDA, J.E.C. de; FILGUEIRA, F.A.R.; CÂMARA, F.L.A. Avaliação de clones de batata-doce em Goiás. Goiânia: EMGOPA-DDT, 1989. 12 p. (EMGOPA, Boletim de Pesquisa, 16).

SILVEIRA, M.A. da; MALUF, W,R. Resistência de clones de batata-doce a Meloidogyne spp. Horticultura Brasileira, Brasília, v. 11, n. 2, p. 131-133, 1993.

STATISTICAL ANALYSIS SYSTEM INSTITUTE. SAS User's Guide. Version 6, Fourth Edition, v. 1, Cary: NY, 1989. 943 p.

THOMAZELLI, L.F.; GANDIM, C.L.; ALMEIDA, E.X.; BOFF, P. Novas tecnologias para o cultivo da batata-doce em SC. Agropecuária Catarinense, Florianópolis, v. 10, n. 1, p. 12-14, 1997. 